\title{
Penicillin sensitivity of gonococci in Australia: Development of Australian gonococcal surveillance programme
}

\author{
MEMBERS OF THE AUSTRALIAN GONOCOCCAL SURVEILLANCE \\ PROGRAMME*
}

SUMMARY We describe the development of the Australian gonococcal surveillance programme from February 1979 to July 1982. Participants in the programme were the major public health laboratories in each state, which perform quantitative sensitivity tests on about 5400 strains of Neisseria gonorrhoeae yearly. Participating laboratories conducted sensitivity tests by a standardised technique which is described, and the results from each centre were collated quarterly. Temporal and regional differences in gonococcal sensitivity patterns were noted and, in the twelve months ending June 1982, there was a trend towards a decrease in gonococci sensitive to penicillin. Increasing numbers of penicillinase producing gonococci were found, and the isolation rates of these strains varied in different regions at different times.

\section{Introduction}

Susceptibility testing of micro-organisms causing clinical infections provides guidance for appropriate antibiotic treatment. In the management of gonorrhoea it is not practicable to delay treatment until the results of susceptibility tests are available, so an alternative approach is generally used. This entails determining the prevailing patterns of gonococcal sensitivities so that the likelihood of a cure in each case is established on an epidemiological rather than an individual basis. ${ }^{1} \mathrm{~A}$ knowledge of prevailing sensitivities of gonococci can also help in the preparation of standardised treatment regimens, ${ }^{2}$ because the response to penicillin treatment in patients infected with Neisseria gonorrhoeae correlates well with the results of in vitro sensitivity testing. Monitoring penicillin sensitivity of gonococci is also valuable in determining the degree of control over gonorrhoea within a community. ${ }^{34}$ Decline in

* W Tapsall (Co-ordinator) and Edna A Phillips, Prince of Wales
Hospital, Randwick, New South Wales; Y M Cossins and D M
Murphy, Laboratory of Microbiology and Pathology, Brisbane,
Queensland; R Mallon and C Macleod, Westmead Hospital,
Wentworthville, New South Wales; J Raby, Woden Valley Hospital,
Canberra, Australian Capital Territory; J R L Forsyth and M M
Peel, Microbiological Diagnostic Unit, University of Melbourne,
Victoria; G Handke and M Lindon, Venereal Diseases Laboratory
of the Institute of Medical and Veterinary Sciences, Adelaide, South
Australia; M Blums and A Ismail, Venereal Diseases Branch
Laboratory, State Health Services, Perth, Western Australia,
Australia.

Address for reprints: Dr J W Tapsall, Department of Microbiology, The Prince of Wales Hospital, Randwick, New South Wales, Australia 2031

Accepted for publication 19 December 1983 the sensitivity of gonococci in an area or region is an indication that gonorrhoea is out of control, whereas a stable low level of resistance is usually evidence of proper management of the disease. Thus for a number of reasons it is desirable to have a knowledge of in vitro sensitivities of gonococci.

The sensitivity of gonococci to antibiotics is determined by agar plate dilution techniques which measure the minimum inhibitory concentration (MIC) of the antibiotic for each strain, and these techniques require careful attention to detail to attain a high degree of accuracy and reproducibility. Another consideration is the fact that different techniques can produce appreciably different results, making it impossible to compare data obtained from different centres. In the continuing gonococcal surveillance programme operating in the United States of America, a central laboratory measures MICs for gonococcal strains from a number of centres. ${ }^{1}$ In Australia, however, each state or territory provides its own health services, and isolates are submitted to one or two large centres in each region. The various state health laboratories in Australia had developed their own techniques for MIC determinations, and therefore truly comparable information on gonococcal sensitivity patterns was not available.

We describe the development of a nationwide surveillance programme for determining the sensitivity of gonococci to penicillin in Australia. Because this country has no large laboratory capable of measuring MICs for all isolates transported to it from other centres, an alternative to the American scheme was explored whereby each participating laboratory used a standardised technique to measure 
the sensitivity of its own isolates. We give details of the methods used, the reasons for their selection, and the results of initial surveys using standardised techniques.

\section{Development of the programme}

Collaborating in this programme were laboratories or their branches which were either attached to public sector sexually transmitted disease (STD) clinics or else received cultures from such clinics. The laboratories concerned were situated in the capital city of each mainland state and in the Australian capital territory. The source of strains, which came from private practitioners as well as STD clinics, and the number of cultures varied little from year to year. The true incidence of gonorrhoea in Australia is unknown, but in 1981 about 12000 clinical cases were notified. About 5400 gonococcal cultures a year are tested for sensitivity to penicillin by the collaborating laboratories.

\section{PHASE I: ASSESSMENT}

Using its own current method each laboratory measured the MIC of penicillin for selected strains of gonococci that were sent in batches of five each month from the coordinating laboratory. These strains comprised WHO reference strains and fresh isolates of gonococci, which had a wide range of MICs. Some strains were duplicated in the same or subsequent batches. There were known differences in techniques between individual laboratories, and this phase of the study assessed how the differences, especially in the use of different media, inocula, and measurement of end points, affected the results. Each laboratory and gonococcal strain was assigned a separate code known only to the coordinating laboratory. Individual laboratories could then identify their own results and assess their performance against results obtained in the other centres.

\section{PHASE II: INTRODUCTION OF A STANDARDISED} TECHNIQUE

During 1980 a standard technique for measuring MICs was proposed and eventually adopted by all of the participating laboratories. The method was based on WHO recommendations $s^{5}$ with the important exception of the medium used. The method was initially circulated in Australia for comment before its adoption, ${ }^{6}$ and is as follows:

\section{Medium}

Isosensitest agar (Oxoid) supplemented with $\mathbf{8 \%}$ horse blood lysed with saponin was used. Penicillin was added to provide final concentrations in the range $0.004 \mathrm{mg} / \mathrm{l}$ to $2 \mathrm{mg} / \mathrm{l}$.

\section{Inoculum}

An inoculum of $10^{4}$ organisms was used. This was obtained by preparing a suspension containing $10^{8}$ colony forming units (cfu) $/ \mathrm{ml}$, as estimated by nephelometry or standard opacity tubes. A 1/100 dilution of this suspension placed in the well of a Steer's type replicating device provided the inoculum. In the coordinating laboratory two further $1 / 100$ dilutions were made, and the Steer's type device was used to deliver three inocula of $10^{6}, 10^{4}$, and $10^{2}$ organisms by using the original suspension and the first two of the serial dilutions. The third and final dilution of the suspension was used to provide a check of the inoculum by means of a surface viable count. These additional procedures were performed in the coordinating laboratory as a continuing assessment of the efficiency of the procedures used. Inoculated plates were incubated in a humidified atmosphere of 5\% carbon dioxide for 18-24 hours at $36^{\circ} \mathrm{C}$. Controls used were the Oxford Staphylococcus aureus (NCTC 6571), WHO reference strains III, V, and VII, and a recently isolated strain.

\section{End point measurement}

The MIC was the lowest penicillin concentration which completely inhibited growth of the $10^{4}$ inoculum. A control plate containing no antibiotic was used for comparison during end point measurements.

The assessment procedures described in Phase I were continued to see how techniques affected results.

PHASE III: COLLECTING AND REPORTING DATA Each laboratory recorded in a uniform way the MIC results obtained using the standardised techniques and made quarterly returns to the coordinating laboratory. In addition, details were recorded of the sex of the patient and the anatomical site from which the isolate was obtained. Strains with an MIC of penicillin in the range of $0.004-0.016 \mathrm{mg} / \mathrm{l}$ were regarded as sensitive, those with an MIC in the range $0.06-0.25 \mathrm{mg} / \mathrm{l}$ as showing decreased sensitivity, and those with an MIC of $>1.0 \mathrm{mg} / \mathrm{l}$ as relatively resistant. For penicillinase producing $N$ gonorrhoeae (PPNG) we tried to ascertain whether the organism was acquired in Australia as a result of sustained domestic transmission or was acquired while the patient was overseas. The external assessment of individual laboratory performance was continued.

\section{Results}

ASSESSMENT PHASE 1979-1980

The range of MICs obtained by participating laboratories for each unknown strain of $N$ gonorrhoeae 
was unacceptably wide. In addition to this variation between laboratories, some laboratories failed to reproduce results when duplicate cultures of a strain were tested on separate occasions. This variation was regarded as unacceptably high if a concentration within one doubling dilution of the original result was not obtained on repeat testing. Typical examples of results obtained in this phase of the study are shown in Tables I and II.

TABLE I MICs of penicillin $G$ for five strains of $N$ gonorrhoeae as measured by six laboratories using varied techniques

\begin{tabular}{ll}
\hline Strain & Range of MICs $(\mathrm{mg} / \mathrm{l})$ \\
\hline A & $0 \cdot 03-0 \cdot 05$ \\
B & $0 \cdot 03-0.05$ \\
C & $0 \cdot 2-1 \cdot 6$ \\
D & $0 \cdot 03-0 \cdot 06$ \\
E & $0 \cdot 01-0 \cdot 1$ \\
\hline
\end{tabular}

TABLE II MICs of penicillin $G$ for one strain of $N$ gonorrhoeae obtained by six laboratories using varied techniques on two separate occasions

\begin{tabular}{|c|c|c|c|c|c|c|c|}
\hline & \multicolumn{6}{|c|}{ MICs (mg/l) obtained by laboratory } & \multirow[b]{2}{*}{ Range } \\
\hline & 1 & 2 & 3 & 4 & 5 & 6 & \\
\hline $\begin{array}{l}\text { 1st test } \\
\text { 2nd test }\end{array}$ & $\begin{array}{l}0.01 \\
0.01\end{array}$ & $\begin{array}{l}0.03 \\
0.01\end{array}$ & $\begin{array}{l}0.03 \\
0.06\end{array}$ & $\begin{array}{l}0 \cdot 03 \\
0 \cdot 12\end{array}$ & $\begin{array}{l}0.06 \\
0.01\end{array}$ & $\begin{array}{l}0.02 \\
0.05\end{array}$ & $\begin{array}{l}0.01-0.06 \\
0.01-0.12\end{array}$ \\
\hline
\end{tabular}

EFFECT OF STANDARDISATION OF TECHNIQUES 1980-1981

Variations between and within laboratories were effectively eliminated during this phase of the study. The MICs obtained for the circulated strains covered a very narrow range, and repeat measurements of the MIC for the same strain fell within the limits of acceptability defined above. Table III shows the results of 21 measurements of the MIC for a single strain in six laboratories using the standardised method. An MIC of $0.06 \mathrm{mg} / 1$ of penicillin was obtained 13 times, and either $0.03 \mathrm{mg} / 1$ or $0.12 \mathrm{mg} / \mathrm{l}$ on the remaining eight occasions. Such results were typical of those obtained in this phase of the study.

TABLE III MICs of penicillin $G$ for a strain of $N$ gonorrhoeae measured 21 times in six laboratories using a standardised technique

\begin{tabular}{llcl}
\hline & \multicolumn{3}{l}{ No of times MICs $(\mathrm{mg} / \mathrm{ll})$ were: } \\
\cline { 2 - 4 } Laboratory & 0.03 & 0.06 & 0.12 \\
\hline 1 & 2 & 2 & \\
2 & 1 & 2 & 1 \\
3 & & 3 & 2 \\
4 & 1 & 3 & \\
6 & & 2 & 4 \\
\hline
\end{tabular}

COLLECTING AND REPORTING DATA 1981-1982 Strains were classified by region and MIC every three months during the year 1 July 1981 to 30 June 1982. These were 1344 isolates tested in the months July to September 1981, 1210 in October to December 1981, 1353 in January to March 1982, and 1448 in April to June 1982.

Figure 1 shows that $80-90 \%$ of strains tested in the first quarter fell into one of two categories, sensitive

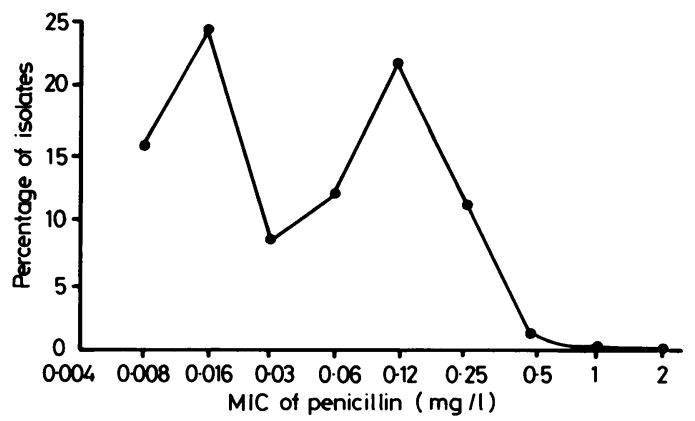

FIG 1 Minimum inhibitory concentrations (MICs) of penicillin for 1344 gonococcal isolates tested from July to September 1981.

or less sensitive to penicillin. Figure 2 shows the distribution by time and region of strains classified by their sensitivity to penicillin. In the first quarter the five centres had a predominance of either sensitive (Perth) or less sensitive (Sydney) strains, or else these two groups of strains were in similar proportions (Melbourne, Adelaide, and Brisbane). During the twelve months of the study this pattern of distribution changed, with an increase in the proportion of less sensitive strains in Perth, Adelaide, and Brisbane. Sensitive and less sensitive strains were isolated in Melbourne in similar numbers throughout the study period. Similarly, there was no change in the relative proportions of the two groups over the twelve months in Sydney, with less sensitive strains remaining predominant. Few non-PPNG strains that were relatively resistant to penicillin were isolated, accounting for only $1-2 \%$ of all cultures.

The percentage of PPNG strains isolated varied between centres and in different quarterly periods. It was consistently high in Perth and low in Melbourne and Adelaide. Brisbane and Sydney isolated increasing numbers of these strains throughout the 12 months. The peak isolation rates of PPNG strains occurred during the Australian summer months in all centres.

The external assessment scheme based on testing for MICs for circulated gonococcal strains gave results similar to those obtained during the previous 12 months. 
Sensitive (MICs $0.004-0.016 \mathrm{mg} / \mathrm{l}$ ) Less sensitive (MICs $0.06-0.25 \mathrm{mg} / 1$ ) Lactamase producers
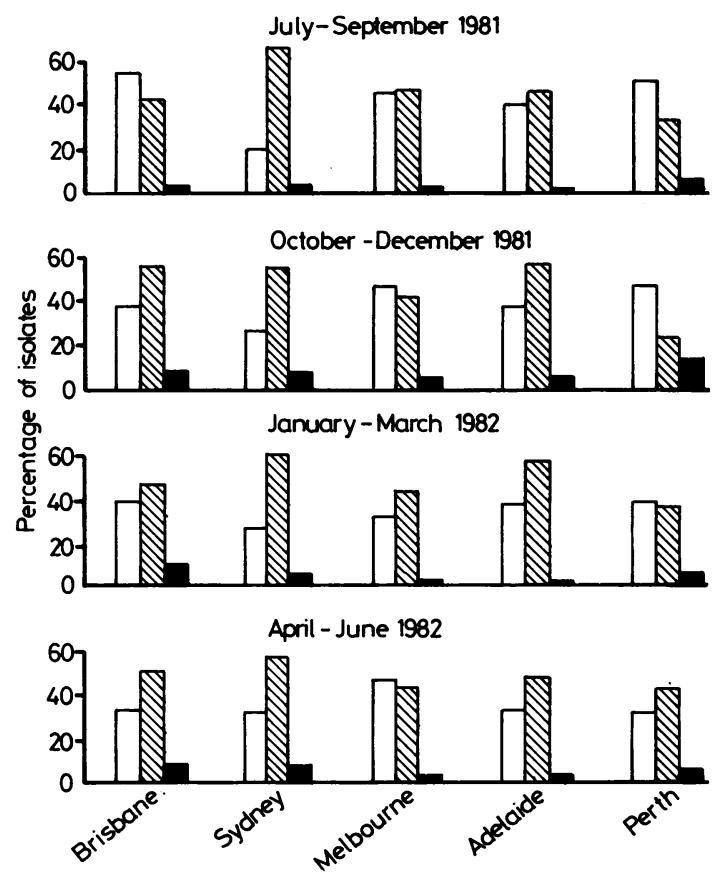

FIG 2 Sensitivity to penicillin of 5355 gonococcal isolates tested in Australia from July 1981 to June 1982 showing quarterly data in five regions.

\section{Discussion}

The Australian gonococcal surveillance programme seeks to obtain national data on patterns of gonococcal sensitivity to penicillin and to compare and contrast regional information within Australia. In this programme, data are derived from results obtained in a number of regional centres. This is a slightly different approach from that in other countries. In the USA, strains from a number of localities are referred to a central laboratory, ${ }^{1}$ and in the United Kingdom national surveillance programmes are concerned with penicillinase producing gonococci. ${ }^{7}$ The initial assessment phase of our programme showed potential problems with the strategy to be adopted. When results from each laboratory were compared there was an unacceptably wide range of results for the selected strains of gonococci because each laboratory was using its own methods. Repeat testing of duplicate strains also produced an unacceptable degree of variation within laboratories. Thus the existing procedures could not have provided valid comparative data on gonococcal sensitivity patterns. Previously published reports on gonococcal sensitivity testing in Australia were not directly comparable because of the diversity of patient samples studied, the intermittent nature of some of the investigations, and the different methods that were used. ${ }^{8-13}$ Similar problems have been acknowledged in some studies in the United Kingdom. ${ }^{14}$ The adoption of a standardised MIC testing procedure effectively eliminated variability between and within laboratories and provided a suitable basis for obtaining information on gonococcal sensitivity patterns in Australia.

The results obtained in the first year of standardised reporting by participants in the programme showed that most isolates were in the sensitive or less sensitive category. This bimodal distribution of results is usual in this type of study. ${ }^{15} 16$ Temporal and regional variations in the proportions of sensitive and less sensitive strains were noted, which could also be expected in such a study. In the USA the national gonorrhoea surveillance programme has been operating for many years, and shifts in sensitivity patterns and variations in these patterns in different regions have been recorded. ${ }^{1}{ }^{15}$ It was noted in Sydney during the 12 months before the introduction of the formal reporting programme that the relative proportions of the sensitive and less sensitive groups had changed. In the period from July 1980 to September 1980 sensitive strains had predominated. In the three subsequent quarterly periods to the end of June 1981 the two groups were in approximately equal proportions, but in the July to September 1981 quarter less sensitive strains predominated for the first time. Although Sydney was the first centre to show this shift, it is noteworthy that all other centres except Melbourne moved towards a predominance of less sensitive strains during the study.

Experience in the USA has also shown that seasonal factors influenced sensitivity patterns, which meant that this type of study needed to be based on a continuing programme. We tested 5355 samples in the 12 months from July 1981 to June 1982. Samples were drawn from the major public STD clinics in each centre so that similar populations were studied in each state. As numbers of samples do not alter significantly from year to year, the results obtained provide a base for continuous comparisons.

The incidence of PPNG strains increased during the study period. The data for PPNG strains in this study include only those lactamase producers identified in or sent to laboratories participating in the programme, rather than the total number of PPNG strains isolated in Australia. Again the source of samples is constant, which allows trends to be detected and regional differences noted. Perth, 
Brisbane, and Sydney had higher incidences of PPNG strains than Adelaide and Melbourne. Countries close to Australia with a high incidence of PPNG strains include the Philippines, Indonesia, Singapore, and Thailand, and the great majority of infections due to PPNG strains detected in Australia are acquired in South East Asia. There is evidence, however, that increasing numbers of PPNG strains detected in Australia have undergone sustained domestic transmission. The reasons for the temporal and regional variation in isolation rates of PPNG strains remain uncertain in the absence of detailed data.

This initial study by the programme participants has shown a trend towards a decrease in sensitivity to penicillin in gonococcal isolates in Australia. It was perhaps fortuitous that the study was conducted during a period when changes in sensitivity patterns were occurring in most of the centres, as this showed that we could detect such changes in Australia. On the basis of the survey it appears that gonococcal disease acquired within Australia is still amenable to treatment with the doses of penicillin in normal use, namely amoxycillin $3 \mathrm{~g}$ plus probenecid $1 \mathrm{~g}$ given as a single dose. This situation could change if the incidence of PPNG strains continues to rise or if sustained domestic transmission of these strains becomes widespread.

\section{References}

1. Jaffe HW, Biddle JW, Thornsberry C, et al. National gonorrhea therapy monitoring study. In vitro antibiotic susceptibility and its correlation with treatment results. N Engl J Med 1976; 294:5-9.
2. Guinan ME, Biddle J, Thornsberry C, Reynolds G, Zaidi A, Wiesner $P$. The national gonorrhea therapy monitoring study: 1. Review of treatment of results and of in vitro antibiotic susceptibility, 1972-1978. Sex Transm Dis 1972:6 suppl:93-102.

3. Jackson DH, Jephcott AE. Penicillin sensitivity of gonococci. An evaluation of monitoring as an index of epidemiological control. Br J Vener Dis 1976;52:253-5.

4. Olsen GA, Lomholt $G$. Gonorrhoea treated by a combination of probenecid and sodium penicillin G. Br J Vener Dis 1969; 45:144-8.

5. WHO Scientific Group. Agar dilution technique to determine sensitivity to antibiotics. WHO Tech Rep Ser 1978 No 616: 137-8.

6. Tapsall JW. Penicillin resistance in $N$ gonorrhoeae. Determination of minimum inhibitory concentrations (MIC) by agar plate dilution techniques. Communicable Diseases Intelligence $1981 ; 4: 3-5$.

7. McCutchan JA, Adler MW, Berrie JRH. Penicillinase-producing Neisseria gonorrhoeae in Great Britain, 1977-81: alarming increase in incidence and recent development of endemic transmission. Br Med J 1982; 285:337-40.

8. Smith DD, Levey JM. Susceptibility of Neisseria gonorrhoeae to penicillin. Med J Aust 1967;1:849-50.

9. Hatos G. Treatment of gonorrhoea by penicillin and a renal blocking agent (probenecid). Med J Aust 1970; 1:1096-9.

10. Tuza FLC, Hatos G. Treatment of male urethral gonorrhoea with spectinomycin hydrochloride (Trobicin). Med J Aus 1973; 2: 1090-1.

11. Smithurst BA. Resistance of Neisseria gonorrhoeae to penicillin in 199 gonococcal infections in women and the response of those infections to penicillin treatment. Med J Aust 1974; 1:585-6.

12. Finger A, Handke G. Antibiotic sensitivity of gonococci in South Australia, 1974 to 1975. Med J Aust 1977; 1:133-6.

13. Thomson KS, Evans DDE. Penicillin resistance of Neisseria gonorrhoeae in Tasmania. Australian Journal of Medical and Laboratory Science 1980; 1:37-9.

14. Herzog C, Ison CA, Easmon CSF. Antimicrobial sensitivity of Neisseria gonorrhoeae: comparison of penicillinase producing and non-penicillinase producing strains. Br J Vener Dis 1983; 59:289-92.

15. Reynolds GH, Zaidi AA, Thornsberry C, Guinan M, Biddle J, Wiesner PJ. The national gonorrrhea therapy monitoring study: II. Trends and seasonality of antibiotic resistance of Neisseria gonorrhoeae. Sex Transm Dis 1972;6 suppl: 103-11.

16. Phillips I, King A, Warren C, Watts B, Stoate MW. The activity of penicillin and eight cephalosporins on Neisseria gonorrhoeae. J Antimicrob Chemother 1976; 2:31-9. 